\title{
Los desastres no son prevenibles si vienen de la ira de Dios
}

\author{
Disasters are not preventable if they come from the wrath of God
}

RESUMEN | Investigación realizada con niños que habían tenido experiencias derivadas de los desastres. Objetivo: Comprender el significado que tienen las situaciones de desastre para los niños de un barrio de la ciudad de Medellín, Colombia. Metodología: investigación cualitativa con enfoque etnográfico, utilizando como técnicas para construir la información: entrevistas, diario de campo y la cartografía social. Hallazgos: los desastres para los niños se originan en la manifestación de la ira de Dios en una población vulnerable. La ira de Dios es por los pecados de los hombres y la vulnerabilidad es debido a las condiciones de sus viviendas y del barrio. Consideraciones finales: Los niños conocen los peligros a los cuales están expuestos, sin embargo no pueden prevenir los desastres porque son causados por la ira de Dios. Se deben elaborar programas de prevención que tengan en cuenta la voz de los niños como participes fundamentales en el cuidado del ambiente.

PALABRAS CLAVE: Desastres. Vulnerabilidad ante desastres. Educación en desastres. Niños.

\section{Paula Andrea Anduquia', Constanza Forero Pulido²} ORCID:0000-0003-4321-7907. paula.anduquia@udea.edu.co de Enfermería. Universidad de Antioquia, Medellín, Colombia. ORCID: 0000-0001-7494-6825. constanza.forero@udea.edu.co

\begin{abstract}
The research was made with children who have been in a natural disaster. Objective: Understand the meaning of a natural disaster for neighborhood's children in Medellin city. Methodology: Qualitative research with ethnographic approach, using interviews, field diary and social cartography to find de information. Research findings: To children a natural disaster is the appearance of God's anger in a susceptible town. God's anger y produced by human sins and the suceptibility produced by the home and neighborhood conditions. Final considerations: Children know the risk of a natural disaster, but they know these are not preventable because are caused by God's anger. To develop a prevention program that have the children's point of view as the main involvement of the environment care.
\end{abstract}

KEYWORDS: Disasters. Disaster vulnerability. Education in disasters. Child. 


\section{Introducción}

Los desastres son consecuencia de fenómenos naturales y antrópicos desencadenantes de procesos que provocan daños físicos y pérdidas de vidas humanas y de capital, al tiempo que alteran la vida de comunidades y personas, y la actividad económica de los territorios afectados. (CEPAL, 2014). Estas situaciones marcan la vida, la cultura, las costumbres de las personas y en consecuencia, la forma de ver el mundo. Un desastre va más allá de la amenaza que lo origina, tiene que ver con el desequilibrio de lo cotidiano donde coexisten el riesgo y su construcción social, la vulnerabilidad, y la resiliencia ante estos eventos.

De manera específica, los niños, pierden la cosmovisión de su entorno como un lugar seguro y predecible cuando viven un desastre. Castillo, Ledo y Toyo (2015). Además, "les producen trastornos importantes por la rapidez con que ocurren los cambios y la manera en que son afectados; los niños sienten miedo, impotencia y aislamiento y esto interfiere en su habilidad para desarrollar un equilibrio funcional con el mundo, alterando su sentido de seguridad y protección" (Lorita, 2010, p. 7).

A sí mismo, El desplazamiento, la separación de los padres y de las redes de apoyo, también el empobrecimiento de familias que ya eran pobres, exponen a los niños a un mayor riesgo de enfermedades relacionadas con la malnutrición, la baja calidad del agua y de saneamiento. Además, el cese de las actividades en la escuela, bien sea por la destrucción de esta o la falta de educadores, aumenta la vulnerabilidad de los niños, exponiéndolos aún más al peligro y el trauma físico y emocional por los eventos vividos. (UNICEF, (2016)

La literatura ha expuesto algunos argumentos de cómo los niños asumen dichos eventos y cuáles son sus respuestas ante los desastres. Estudios como el realizado por Zhang et al., (2010), determinaron que los eventos de origen natural como los terremotos donde las pérdidas humanas y materiales son mayores, el afrontamiento de los infantes es más complejo, generando alteración en sus procesos mentales y comportamentales. En contraste, autores como Cryder, Kilmer, Tedeschi y Calhoun (2006) refieren que las creencias y competencias positivas inculcadas en los niños, cambia significativamente la forma como estos perciben y afrontan los desastres.
Por su parte, Tanner, Rodríguez y Lazcano (2008) muestran el potencial de los grupos juveniles como agentes de cambio en sus comunidades, y aportan una llamada para la participación activa de los niños y jóvenes en la gestión de riesgos para prevenir los desastres. Además, argumentan que los niños han desarrollado importantes habilidades de comunicación en la prevención del riesgo, lo cual deber ser aprovechado para incidir en el hogar, en la escuela y en aquellos espacios comunitarios donde los niños y niñas han encontrado un escenario de participación.

En nuestro medio se desconocen estudios realizados desde la mirada de los niños que permitan lograr los cambios esperados en la construcción de la cultura del cuidado ambiental. Por lo tanto, con este trabajo se buscó comprender el significado que tienen las situaciones de desastre para los niños de un barrio de la ciudad de Medellín, Colombia. Esta investigación permitirá generar conocimiento sobre los niños como participantes activos en los procesos de la gestión del riesgo y como multiplicadores del cambio en los comportamientos sociales frente al cuidado del ambiente.

\section{Metodología}

Esta investigación es de tipo cualitativo etnográfico. Como se pretendió comprender significados y acciones, desde la mirada de los participantes, se utilizó la etnografía porque permitió describir y analizar los procesos culturales dentro de un contexto, definido como aquel conjunto de significados encarnados en símbolos; concepto fundamental de esta perspectiva. (Geertz, 2003).

El etnógrafo, en su condición de investigador, no parte de conocimientos previos y por lo tanto debe iniciar un proceso de conocimiento, por medio del trabajo de campo, es decir, construye el conocimiento con base en la interacción que establece con el grupo observado. El investigador pasa de la mirada al sentido, como afirma Galindo (1998). Según Boyle (1994), la conducta de las personas sólo puede ser entendida en el contexto, que implica más que el ambiente físico, los procesos de análisis y abstracción.

Los participantes fueron 24 niños escolarizados entre los 7 y 12 años de edad habitantes de un Barrio del 
Municipio de Medellín Colombia, que estuvieron presentes en una situación de desastre sin importar su origen; se indagó sobre experiencias vividas por ellos con relación al evento.

Se hizo un acercamiento con las líderes comunitarias y los padres de familia de los niños que participaron en la investigación, con el propósito de exponer los objetivos, metodología y técnicas; así mismo, se les informó acerca de su libre participación durante el proceso. Una vez se obtuvo el consentimiento de los padres de forma verbal y escrita, se habló con los niños para obtener su asentimiento y concertar las fechas y horas para llevar a cabo los encuentros, las actividades y las entrevistas. Las sesiones fueron realizadas por los investigadores, grabadas y posteriormente transcritas y las actividades lúdicas fueron elaboradas de acuerdo con las edades de los participantes

Se llevaron a cabo 6 encuentros grupales y se desarrollaron 8 actividades con una duración de dos horas aproximadamente por cada sesión. El propósito de la primera actividad fue propiciar un espacio de acercamiento de los participantes con la investigadora y la temática del trabajo a través de un juego de integración.

En las sesiones posteriores, se trabajaron temas como la conceptualización de los desastres, la identificación de las amenazas de su entorno partiendo de sus experiencias y conocimiento previo, el origen y consecuencia de los fenómenos que causan el desastre y finalmente, qué acciones realizan los niños y niñas cuando se presenta un evento natural.

Igualmente, se utilizaron técnicas como la entrevista abierta, el diario de campo y la observación y se realizaron hasta lograr la saturación de la información, entendida esta como el punto en el cual ya no emergieron propiedades, dimensiones, o relaciones nuevas durante el análisis.

La observación se realizó en el barrio haciendo recorridos con la comunidad, previo acercamiento con los líderes y con los niños que participaron en el estudio para conocer las condiciones en las que vivían.

La cartografía social, permitió el acercamiento al territorio, el desarrollo de un lenguaje común de interpretación de las diferentes percepciones de la realidad y la recolección de información vivencial por medio de la construcción de mapas de amenazas o peligros, Juegos de integración, lluvia de ideas y la elaboración de cuentos donde los niños expresaron las experiencias entorno al desastre y sobre lo que ocurre en su comunidad cuando las amenazas se materializan. Los dibujos elaborados permitieron identificar los lugares donde se ubican los peligros tanto en sus hogares como en el barrio y las puestas en escena evidenciaron las actividades que los participantes realizaron ante un evento desastroso.

Con respecto al análisis de la información, este fue un proceso continuo que permitió organizar los datos obtenidos durante la investigación y darle sentido a los significados que emergen de las experiencias y conocimientos de los niños acerca de los desastres. Este se hizo en la medida en que se recolectaba la información a partir de la lectura de las entrevistas transcritas, los registros obtenidos del diario de campo, las observaciones realizadas y las construcciones de la cartográfica social. La codificación de la información y posterior categorización, se realizaron de forma manual, con asignación de colores para cada una de las categorías y la identificación de cada uno de los niños con nombres de personajes elegidos por ellos. Se realizaron en varias oportunidades lecturas de las entrevistas y lo expresado por los niños en la recolección de la información, luego se fragmentaron los datos en unidades de sentido para obtener códigos, posteriormente se agruparon para obtener la categoría central y las subcategorías; el análisis se hizo a la par de la obtención de los datos, teniendo en cuenta "el carácter emergente de los datos" (Velasco \& Díaz de Rada, 2004).

La información fue plasmada en un mapa conceptual con el propósito de tener un planteamiento general de la información y posteriormente realizar la descripción de los códigos, categorías y subcategorías y como se relacionan entresí. Una vez obtenida esta categorización, se llevó a cabo la teorización, en la cual se plasmaron los testimonios de los niños y se contrastó con la revisión bibliográfica consultada y la argumentación de algunos autores sobre la gestión del riesgo y la participación de los niños en este proceso social.

Los criterios de rigor según Castillo y Vásquez (2003) se logran por: la credibilidad y la auditabilidad. En la investigación estos aspectos se obtuvieron mediante la confirmación de los hallazgos y su revisión con los participantes, la utilización de grabaciones magnetofónicas. Todas actividades fueron grabadas para obtener mayor fidelidad y se recolectaron los 
datos en diferentes momentos, posterior a ello se transcribieron a la mayor brevedad posible para de esta manera obtener la información y dar cuenta de las experiencias vividas por los niños, se tuvo en cuenta siempre su punto de vista, se respetaron sus expresiones. Los resultados se contrastaron en forma permanente con expertos, técnicas y bibliografía,

\section{Consideraciones éticas}

Las directrices éticas tenidas en cuenta dentro de la investigación, se fundamentaron en el respeto y reconocimiento de los participantes. Se garantizó la confidencialidad de la información obtenida; los datos se analizaron de manera global y la información se obtuvo de manera impersonal utilizando seudónimos que los niños seleccionaron, las entrevista se guardaron en el archivo de una de las investigadoras y en ellas no aparecen nombres.

El consentimiento informado se obtuvo de forma verbal y escrita del representante legal del niño y del niño participante según lo establece el artículo 16 de la Resolución 8430 de 1993 del Ministerio de Salud de Colombia. Se solicitó autorización para la grabación de las entrevistas, todos aceptaron gustosamente y sin coartar su decisión de retirarse en cualquier momento. No se utilizó ningún tipo de presión directa o indirecta para que se vincularan a la investigación.

La investigación no requirió de procedimientos de intervención ni experimentales por lo que no implicó riesgos para los participantes. Los investigadores no tenían conflicto de intereses y fue aprobada por el Comité de Educación de la Escuela de Ingenieros Militares.

\section{Hallazgos}

Para los niños los adultos cometen pecados que ocasionan la ira de Dios, esta se manifiesta por medio de fenómenos naturales que ocasionan desastres a la población en un barrio que está ubicado una zona vulnerable, causando daños severos en las casas, familia y entorno. Estas situaciones no se pueden prevenir porque no es posible intervenir en el comportamiento de los adultos.

\section{Descripción del barrio}

El barrio, es un asentamiento suburbano poblado por las familias desplazadas de la región de diferentes subregiones del departamento de Antioquia . Las viviendas, están construidas con adobe y madera, techos de zinc y materiales reciclables, cuenta con una sola vía de acceso y la red de servicios como el agua potable y la energía solo está disponible para la zona baja del asentamiento; la parte alta, debe suplir la red de servicios con agua del acueducto comunitario que se sustenta en dos quebradas, esto se realiza por medio de mangueras desde el tanque comunitario o desde el mismo nacimiento, de igual modo el alcantarillado se encuentra con técnica de cielo abierto.

El suelo de la ladera donde está ubicado el barrio es quebradizo, con fallas geológicas naturales lo que la hace zona con características variables como zonas de riesgo mitigable, zonas de riesgo recuperable y no recuperable. La tierra presenta condiciones arcillosas, áridas y rocosas de condiciones poco aptas para el cultivo. Sin embargo, las personas que habitan es esta zona, sostienen cultivos de plátano y hortalizas, así como de maíz y yuca para su propio sustento y para el intercambio con sus vecinos. (Ortiz, 2012: p.7)

Al urbanizar laderas cada vez más altas y periféricas y zonas más próximas a las quebradas, especialmente en los extremos oriental, nororiental y noroccidental de la ciudad, los desastres asociados a deslizamiento se inundaciones se hicieron más recurrentes. (Serna, 2011).

Detrás de la casa hay un barranco y ya se ha derrumbado y con el agua puede que se derrumbe" (Duque, 7 años)

\section{Causa de los desastres}

Para los niños los desastres causan daños y los definen como un evento que destruye sus casas y que pueden ocasionarle la muerte a su familia, conformada por la mamá y los hermanos.

"Es algo que puede dañar muchas cosas, una catástrofe donde mueren las personas" (Maluma, 12 años) 
El desastre se asocia con Dios quien como ser supremo se enoja cuando se cometen pecados, esto por lo tanto se debe pagar. Esta ira de Dios para los niños se manifiesta con sismos, vientos y lluvia, que hacen muchos daños por presentarse en el barrio donde ellos viven y que reconocen que es una zona vulnerable.

Frente a los eventos enviados por Dios no se pueden realizar actividades de prevención o mitigación para protegerse y proteger a su familia porque los niños no pueden impedir los pecados de los adultos

\section{Manifestaciones de la ira de Dios}

El sismo como manifestación de la ira de Dios, genera un movimiento de tierra que afecta las columnas, el piso, los muros y los caminos al desprenderse la estructura, la tierra y las piedras; hace que la casa se caiga por las características de las construcciones que consideran débiles e inestables, algunas se ven deterioradas especialmente en el piso y además, la tierra que las sostiene, en ocasiones es removida por los adultos porque todo está fabricado de forma artesanal.

"Tiene mucho peligro porque los palos están podridos y la tierra se va derrumbando" (Vale, 8 años)

"El peligro es que una columna de la casa está más gorda que la otra y como escarbaron y la otra esta flaca se le ven las varillas porque esta picada y hay unos huecos" (James Rodríguez, 10 años)

Los vientos generan fuerzas tan fuertes que arrancan los techos construidos en su mayoría de zinc, latas, tejas de barro y plástico y por ser débiles se desplazan destechando las casas dejándolas descubiertas y a las personas y enseres expuestos a los daños generados por la caída de pedazos de material

"Se lleva las tejas cuando ventea mucho y ahí duerme mi hermanita chiquita" (Frozen, 7 años)

La lluvia es la manifestación de la ira de Dios que más se presenta, inicialmente se anuncia por sonidos, luego se desata la lluvia que arrastra primero con el lodo que cubre las calles por donde transita la población, luego con las piedras que se han utilizado para sostener algunas construcciones, finalmente se lleva lo que encuentra a su paso, entra a las casas y las inunda. La tierra de los barrancos ubicados en la parte posterior de las viviendas se humedece, generando desprendimiento de la tierra y piedras que caen sobre los techos y muros destruyendo la casa.

"Lo que me da miedo de mi casa es que llueva y la tierra se ponga húmeda y se caiga" (Ben 10, 8 años)

En los periodos lluviosos por la alta precipitación, las quebradas aumentan su caudal y como en el barrio hay dificultades en el manejo de residuos que muchas veces terminan en las quebradas, el agua se represa y se sale generando inundaciones en el barrio.

"La quebrada, se mantiene llena de basura y se inunda; ya el agua se entró a la casa una vez." (Maluma, 12 años)

\section{Discusión}

La gestión del riesgo es una construcción social y cultural partiendo de las experiencias de las comunidades, los riesgos que ya existen y los que a futuro pueden afectarlos. No es solo la identificación y estudio de las amenazas, de la vulnerabilidad y la reducción del riesgo; implica, además, la participación de la comunidad en la toma de decisiones y las actividades que como colectivo pueden realizar para prevenirlos.

Los niños y niñas como participes en el proceso de la gestión del riesgo, construyen sus propios significados sobre los desastres a partir de las experiencias e imaginarios. Para ellos, los desastres los causa la ira de Dios como consecuencia de los pecados del adulto; este significado, hace que la vulnerabilidad aumente al limitar las acciones de acuerdo a sus capacidades para prevenir y reducir los factores relacionados con el riesgo.

En estas condiciones y partiendo de la premisa que los niños y niñas participantes de este estudio tienen conocimientos básicos relacionados con los desastres, se esperaría que tuvieran presentes las medidas y acciones que permitieran evitar la materialización de los peligros o aquellas encaminadas a la reducción de los efectos en la comunidad, las viviendas, servicios y el medio ambiente. Sin embargo, el concepto que tienen de Dios y su relación con la manifestación de los desastres, no les permite comunicar y sensibilizar 
a sus familias y a la comunidad sobre los riesgos y el desastre. Dios es quien decide cómo y cuando llegan los fenómenos que causan los desastres y como se manifiestan, es Él quien decide con su voluntad, cuando se puede actuar y cuando no.

\section{La ira de Dios}

Los desastres son circunstancias de la vida diaria con las cuales el hombre convive desde su origen; las consecuencias derivadas de estos sucesos han sido medidas en pérdidas humanas y materiales a corto y mediano plazo. Sin embargo, por sus repercusiones a todo nivel y el impacto en el proceso histórico de las comunidades, deben ser considerados como fenómenos sociales multicausales, complejos y continuos, analizados desde varias miradas e intervenidos de manera holística.

Una de estas miradas, concibe el origen de los desastres como castigo de Dios, esta concepción se encuentra consignada en los escritos bíblicos como en el Génesis (1:26-27), donde se enuncia que Dios hizo a los seres humanos a su imagen y les dio dominio sobre el mundo natural, los separó del resto de la creación como únicos y como representantes de Dios habían de reinar sobre la naturaleza tal como Él lo había proyectado. En Romanos, (5:12 y 8:20), relatan que una vez que el pecado y el mal entraron en el mundo, todo cambió. Los humanos y la naturaleza ahora están bajo la carga del pecado y del mal (vers.22).

Bauman (2007), también afirma, que el mal nació con el propio acto del pecado y que era devuelto a los propios pecadores en forma de castigo. Caballero (2007) plantea que esta concepción está motivada por las malas acciones o pecados y que permite pensar que la maldad es digna de castigo colectivo. En palabras de Rodríguez (2008), "los desastres Ilamados como naturales, han de ser esperables en un mundo de pecado y de mal. No son la excepción a la regla, sino la regla misma. Inusual es que no haya más de ellos. La presencia "discontinua" de los desastres naturales nos dice que Dios aún está al control, sustentando y preservando la naturaleza." (p.3)

Peñalta (2009), contempla que "todos los males que afectan al hombre forman parte de un sistema, que todo ocurre para bien y por disposición de Dios". En una investigación realizada en la Isla de Granada y posterior al paso del Huracán Iván, destaca como los niños de acuerdo a sus experiencias y la percepción sobre el desastre, señalan a Dios como uno de los primeros factores, incluso como una explicación de la razón por la cual ocurrieron los huracanes. Expresaban que un huracán es un fenómeno de la naturaleza, pero es Dios quien los envía a la tierra. (Lorita 2010, p.15)

En este sentido, cuando el significado que se le da al origen de los desastres es visto desde lo divino y no como un desequilibrio del sistema naturalezaser humano, afloran otras explicaciones que limitan las acciones para intervenir en la prevención y mitigación de los desastres. Según Romero y Maskrey (1993), estas nuevas justificaciones, promueven en las personas sentimientos de dolor, espera pasiva y resignación que dificulta más su reparación después de un desastre y aumenta su percepción de vulnerabilidad.

Es así como la vulnerabilidad es un problema global en el que intervienen aspectos sociales, culturales, políticos, y ambientales, que interactúan con el entorno, los recursos naturales, las personas y sus necesidades, que en consecuencia condicionan su bienestar, seguridad, calidad de vida y proyección hacia el futuro. En este sentido, la vulnerabilidad y sus efectos, van a depender de la destrucción que puede causar una amenaza, de la preparación para responder cuando el peligro se materializa y de las capacidades de la población y de la naturaleza para retornar a sus condiciones de vida previas o mejorarlas.

Peralta (2013) describe la vulnerabilidad como el resultado de las necesidades insatisfechas de la sociedad moderna, que se asocia a los modelos de desarrollo imperantes, y se materializa en el entorno construido. El grado de vulnerabilidad física y funcional de una población, dependerá del grado de satisfacción o insatisfacción de las necesidades sociales, asociadas a las dimensiones del desarrollo y que se podrían expresar como una sumatoria de factores en la que unos $u$ otros pueden tener mayor peso o relevancia.

Esto concuerda con lo encontrado en esta investigación, al relacionar la vulnerabilidad con la insatisfacción de las necesidades básicas. Los niños que hacen parte de este asentamiento subnormal ubicado en zona de alto riesgo, deben sobrevivir en condiciones de precariedad, con servicios públicos 
deficientes, en condiciones de pobreza abrumadoras, viviendas técnicamente mal construidas y con difícil acceso a la salud y educación.

Los niños reconocen que viven en una zona de alto riesgo, que las amenazas a las cuales están expuestos destruyen sus viviendas, causa muerte a su familia y que altera las dinámicas del barrio. Sin embargo, su vulnerabilidad aumenta al expresar que no pueden actuar porque los desastres son un castigo de Dios que se manifiesta para saldar los pecados que el adulto comete y que todos deben pagar por ello.

Esto demuestra el poco acompañamiento por parte de las instituciones que trabajan en la gestión del riesgo en la ciudad; además, las acciones y estrategias que utilizan para gestionar el conocimiento no son efectivas porque no escuchan a la población y mucho menos a los niños sobre los significados que tienen los desastres para ellos, el por qué se producen y que acciones deben realizar para prevenirlos 0 mitigarlos. Si se escucha a la población y se parte de sus experiencias previas y conocimientos, la gestión del riesgo en comunidades vulnerables será más consciente en tanto son tenidos en cuenta, los hacen participes de su protección, líderes en la transformación social y el cuidado del ambiente.

\section{Consideraciones finales}

Los niños definen los desastres como manifestaciones de la ira de Dios por los pecados que el adulto comete.

El significado que tienen los niños sobre los desastres y su origen, no les permite realizar actividades de prevención y sensibilización sobre los riesgos y los desastres. Dios es quien decide cómo y cuándo se manifiestan los peligros y ante esto no se puede hacer nada.

Los niños conocen los peligros a los cuales están expuestos, reconocen las medidas de mitigación que ejecutan los adultos; sin embargo, dichas medidas no disminuyen la vulnerabilidad, pero sí, agrava las situaciones de riesgo.

El trabajo de campo permitió un acercamiento con los niños y la posibilidad de presentar la visión de ellos. Este trabajo es la voz de los participantes relacionada con lo que sienten y por lo tanto se pueden hacer recomendaciones para elaborar programas de gestión de riesgo. Realizar más investigaciones desde la mirada de los niños que contribuyan a mejorar las condiciones del planeta y que favorezca su calidad de vida.

Es necesario elaborar programas educativos acerca de la identificación de los factores de riesgo que originan los desastres, que permitan deconstruir las atribuciones Divinas y construir significados que propicien la prevención y cómo actuar en caso que se presenten.

\section{Agradecimientos}

A la Facultad de Enfermería de la Universidad de Antioquia y a la Escuela de Ingenieros Militares, Por el apoyo para desarrollar este trabajo.

Este artigo é resultado de pesquisa aprovada para apresentação no $7^{\circ}$ Congresso Ibero-Americano em Investigação Qualitativa - CIAIQ, realizado de 10 a 13 de julho de 2018 em Fortaleza, Ceará, Brasil.

\section{Contribuições das autoras}

Anduquia, P. A. participou da elaboração do desenho de estudo, preparação, coleta de informações, interpretação e redação de resultados e discussão; e escrita do de artigo científico.

Forero, C. participou do projeto, preparação, coleta de informações, informações e redação de resultados e discussão; e escrita do artigo científico.

\section{Conflitos de interesses}

Nenhum conflito financeiro, legal ou político envolvendo terceiros (governo, empresas e fundações privadas, etc.) foi declarado para nenhum aspecto do trabalho submetido (incluindo mas não limitando-se a subvenções e financiamentos, participação em conselho consultivo, desenho de estudo, preparação de manuscrito, análise estatística, etc.).

\section{Bibliografía}

Bauman, Z. (2007). Miedo líquido. La sociedad contemporánea y sus temores. Barcelona: Paidós.

Buriticá, H. A. P., Peñaloza, A. H., Herrera, F. E. (2013). Territorios resilientes. Guía para el conocimiento y la reducción del riesgo de desastre en los municipios Colombianos. Bogotá: Federación Colombiana de Municípios. 
Caballero, J. H. (2007). La percepción de los desastres: algunos elementos desde la cultura. Gestión y Ambiente, 10(2), 109-115. Recuperado de http://www.redalyc.org/articulo. oa?id=169419816009

Cáceras, J. G. (1998). Etnografía: El oficio de la mirada y el sentido. In J. G. Cáceras. Técnicas de Investigación en Sociedad Cultura y Comunicación. México D.F: Ed Adison, Wesley, Longman.

Castillo, E. Vásquez, M. L. (2003). El rigor metodológico en la investigación cualitativa. Revista Colombia Médica, 34(3), 164-167. Recuperado de: http://www.redalyc.org/ pdf/283/28334309.pdf

Catalán, R. P. (2009). Voltaire: “Una reflexión filosófico-literaria sobre el terremoto de Lisboa de 1755. Revista de Filología Románica, 26, 187-204. Recuperado de http://revistas.ucm. es/index.php/RFRM/article/view/RFRM0909110187A/9225

CEPAL (2014). Manual para la evaluación de desastres. Recuperado de https://repositorio.cepal.org/bitstream/ handle/11362/35894/1/S2013806_es.pdf

Cryder, C. H., Kilmer, R. P., Tedeschi, R. G., \& Calhoun, L. G. (2006). An exploratory study of posttraumatic growth in children following a natural disaster. American Journal of Orthopsychiatry, 76(1), 65-69. Recuperado de https:// psycnet.apa.org/fulltext/2006-03377-010.html. doi: 10.1037/0002-9432.76.1.65

Geertz, C. (2003). La interpretación de las culturas. Barcelona: Editorial Gedisa.

Giraldo, A. O. (2012). Caracterización Comuna 3 Manrique de la Ciudad de Medellín. Recuperado de https://www.kavilando. org/images/stories/documentos/CARACTERIZACION_ COMUNA3_MANRIQUE_O_G.pdf

Ledo I. C., González, H. L., Calderin, Y. T. (2015). Repercusión de los desastres en la salud mental de niños/as y adolescentes. Norte de salud mental, 13(53), 26-34. Recuperado de https://dialnet.unirioja.es/servlet/articulo?codigo $=5265701$

Lorita, J. (2010). Exploración de las percepciones infantiles ante desastres naturales (huracanes): estudio de niños en Granada. Revista Trabajo Social, (78) ,7-16. Recuperado de https://repositorio.uc.cl/handle/11534/6535

Quintana, C. A. S. (2011). La naturaleza social de los desastres asociados a inundaciones y deslizamientos en Medellín (1930-1990). Historia Crítica, (43), 198-223. Recuperado de http://www.scielo.org.co/pdf/rhc/n43/n43a11.pdf
Resolucion $n^{\circ} 8430$, de 4 de Octubre de 1993. Por la cual se establecen las normas científicas, técnicas y administrativas para la investigación en salud. Recuperado de https://www.minsalud.gov.co/sites/rid/Lists/ BibliotecaDigital/RIDE/DE/DIJ/RESOLUCION-8430-DE-1993. PDF

Rodríguez, A. M. (2008). Dios y la Naturaleza. Recuperado de https://pt.scribd.com/document/287979528/AMRodriguez-dios-y-La-Naturaleza

Romero, G., \& Maskrey, A. (1993). Como entender los desastres. In A. Maskrey (Ed.), Los desastres no son naturales (pp. 1-9). Colombia: La Red.

Tanner, T., Rodríguez, G., \& Lazcano, J. (2008). Los niños y niñas y la gestión de riesgos: Un rol clave en la prevención de desastres. Medio Ambiente y Urbanización, 69(1), 117133. Recuperado de https://www.preventionweb.net/ files/7859_s9.pdf

UNICEF (2016). Reducción del riesgo de desastres centrada en la niñez: contribuir al desarrollo resiliente. Recuperado de https://www.unicef.org/lac/informes/reducci\%C3\%B3ndel-riesgo-de-desastres-centrada-en-la-ni\%C3\%B1 ez

Vásquez, P. A. A., \& Pulido, C. F. (2018). Significado del desastre para los niños de la comunidad de la Honda Medellín. Atas CIAIQ2018 - Investigação Qualitativa em Ciências Sociais, Fortaleza, CE, Brasil. Recuperado de https://proceedings. ciaiq.org/index.php/ciaiq2018/article/view/1715/1668

Velasco, H., \& Rada, A. G. (2004). La lógica de la inevstigación etnográfica. Un modelo de trabajo para etnógrafos de escuela (4 ed). Madrid: Trotta.

Zhang, Y., Kong, F., Wang, L., Chen, H., Gao, X., Tan, X., ... Liu, Y. (2010). Mental health and coping styles of children and adolescent survivors one year after the 2008 Chinese earthquake. Children and Youth Services Review, 32(10), 1403-1409. doi: 10.1016/j.childyouth.2010.06.009 